Cinémas

Revue d'études cinématographiques

Journal of Film Studies

\title{
Marques d'un cinéma moderne : Le Roi des enfants
}

\section{Dominique Brochu}

Volume 3, numéro 2-3, printemps 1993

Le nouveau cinéma chinois

URI : https://id.erudit.org/iderudit/1001189ar

DOI : https://doi.org/10.7202/1001189ar

Aller au sommaire du numéro

Éditeur(s)

Cinémas

ISSN

1181-6945 (imprimé)

1705-6500 (numérique)

Découvrir la revue

Citer cet article

Brochu, D. (1993). Marques d'un cinéma moderne : Le Roi des enfants. Cinémas, 3(2-3), 8-22. https://doi.org/10.7202/1001189ar
Résumé de l'article

$\mathrm{Au}$ sortir du règne maoïste, le mouvement du nouveau cinéma chinois cherche à trouver son indépendance : indépendance de l'art et de l'artiste, indépendance du contenu et de la forme. Les marques repérées dans le film $L e$ Roi des enfants (1986) de Chen Kaige démontrent l'actualisation de cette démarche réflexive à travers un langage propre au cinéma moderne. Se juxtapose alors une double lecture du film. Le niveau cinématographique et le niveau narratif proposent un même cheminement par leur distanciation et leur questionnement face à la tradition. 


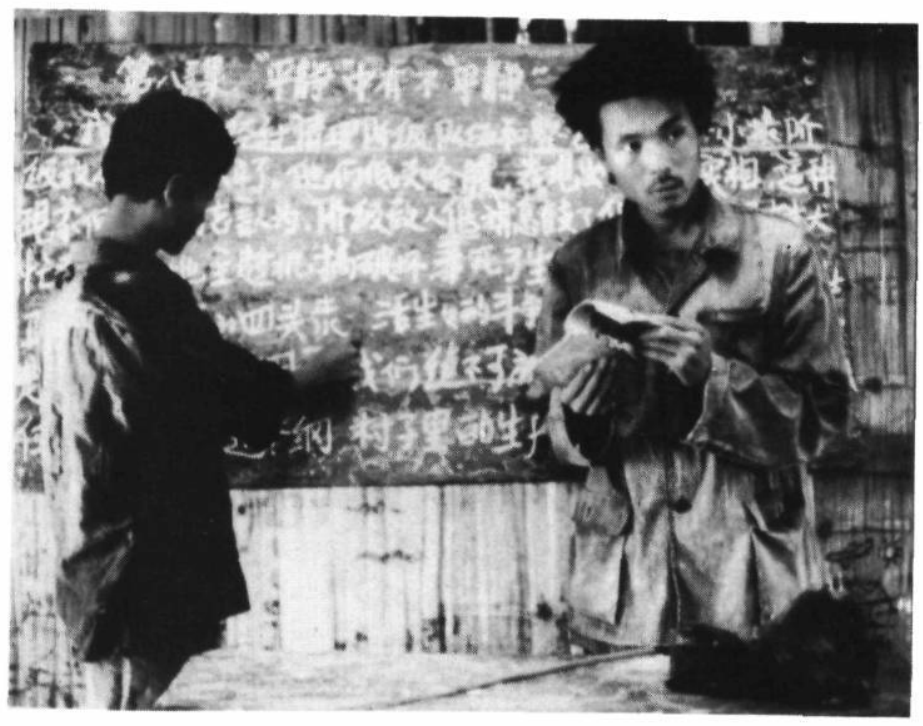

Le Roi des enfants de Chen Kaige (1986) 


\title{
Marques d'un cinéma moderne: Le Roi des enfants
}

\section{Dominique Brochu}

\begin{abstract}
RÉSUMÉ
Au sortir du règne maoïste, le mouvement du nouveau cinéma chinois cherche à trouver son indépendance : indépendance de l'art et de l'artiste, indépendance du contenu et de la forme. Les marques repérées dans le film Le Roi des enfants (1986) de Chen Kaige démontrent l'actualisation de cette démarche réflexive à travers un langage propre au cinéma moderne. Se juxtapose alors une double lecture du film. Le niveau cinématographique et le niveau narratif proposent un même cheminement par leur distanciation et leur questionnement face à la tradition.
\end{abstract}

\section{ABSTRACT}

At the end of the Maoist period, the new cinema movement in China sought to regain its independence : independence of art and of the artist, of content and of form. Manifestations of this reflexive process found in the film King of the Children (1986) by Chen Kaige show how it was expressed by means of the particular language of modern cinema. The film thus receives a double reading : the cinematographic and narrative levels follow the same course in distancing themselves from tradition and inciting a questioning of the self in relation to tradition.

À l'aube des années 80 , les réformes et l'ouverture de la Chine ont permis au cinéma chinois de s'ouvrir sur le monde. Le nouveau cinéma chinois né de ces réformes n'est pas seulement la manifestation d'une simple volonté de modernisation 
technologique, il est aussi la manifestation du désir de sortir de l'isolement pour participer, comme partout dans le monde, au développement du cinéma dans son ensemble. La participation et les différents prix obtenus par les Chinois aux différents festivals internationaux marquent l'entrée du cinéma chinois, et surtout sa reconnaissance, dans la communauté cinématographique internationale. Les spécialistes tant chinois qu'étrangers perçoivent dans le nouveau cinéma chinois une certaine maturité cinématographique grâce à une bonne combinaison des techniques occidentales et de la qualité de l'expression de la culture chinoise.

Pour cette génération de réalisateurs, le cinéma se pose effectivement à l'intérieur de cette double problématique : réfléchir sur une esthétique qui soit à la fois spécifique à l'art cinématographique et au reflet d'une culture. Nous nous proposons donc de consacrer cet article à identifier les marques de cette démarche réflexive à l'intérieur d'une des œuvres du nouveau cinéma chinois. Le Roi des enfants, réalisé par Chen Kaige en 1986, nous semble le film le plus représentatif et peutêtre le plus achevé parmi la production chinoise de la Cinquième génération entre 1982 et 1989.

Jean Baudrillard définit la modernité de la façon suivante :

Un mode de civilisation caractéristique, qui s'oppose au mode de la tradition, c'est-à-dire à toutes les autres cultures antérieures ou traditionnelles (...). Comme elle n'est pas un concept d'analyse, il n'y a pas de lois de la modernité, il n'y a que des traits de la modernité. Il n'y a pas non plus de théorie, mais une logique de la modernité et une idéologie. Morale canonique du changement, elle s'oppose à la morale canonique de la tradition, mais elle se garde tout autant du changement radical1.

Le détachement envers une tradition provoque nécessairement le questionnement et la recherche de sa propre essence. Ce qui marque l'ère moderne, c'est la capacité de l'homme à se distancier face au monde qui l'entoure et à réfléchir sur l'homme et sur sa façon d'appréhender le monde. Ainsi, le cinéma moderne sait prendre ses distances du cinéma traditionnel et il sait réfléchir sur sa propre condition ainsi que sur sa forme expressive. En accord avec cette logique, nous avons tenté de retrouver dans Le Roi des enfants les traits caractéristiques de cette idée du modernisme.

Le scénario du film vient d'un roman du même titre de A Cheng. Il s'agit du récit d'un jeune instruit (zhi qing) pendant la Révolution culturelle; il travaille sur un chantier de coupe de bois. Les autorités du canton l'assigne à une école primaire pour enseigner à un groupe d'élèves de dernière année. Dès son premier jour de classe, il découvre l'unique exemplaire d'un seul 
livre qu'il devra faire copier mot à mot par ses élèves. Après la copie des premières leçons, l'instituteur réalise que les élèves ne comprennent pas les caractères qu'ils transcrivent pourtant depuis des années. Cette découverte le portera à réfléchir sur le rôle de l'enseignement. Il prend la décision de ne plus employer ce livre scolaire à fort contenu idéologique. Par le biais de compositions, le professeur incitera les enfants à formuler leurs propres pensées, à ne plus copier les idées des autres afin de tirer des leçons de leur propre expérience.

\section{Le refus du drame comme structure narrative}

L'une des marques les plus évidentes du cinéma moderne consiste peut-être en ce refus de la tradition d'un cinéma s'appuyant sur le récit dramatique. Le cinéma classique construit un film en fonction d'une histoire. Comme en Occident, le cinéma chinois s'est inspiré du modèle théâtral en privilégiant le drame comme structure narrative.

Le Roi des enfants ne se détache pas complètement de cette tradition, car il y a toujours récit. Par ailleurs, Baudrillard indique bien de la modernité cette mise en garde d'un changement radical, tendant en faveur d'un amalgame de la tradition et du moderne. Ainsi, le film s'éloigne de la tradition en évitant du récit sa mise en forme dramatique.

Le film rompt également avec la tradition cinématographique chinoise. À l'inverse du mélo, du discours flamboyant et de l'action héroïque, Le Roi des enfants peut nous laisser une impression d'inaction et d'extrême lenteur. La narration réduit l'intrigue à son minimum. Le récit se déroule en un rythme lent et régulier. Il n'y a pas de rupture de rythme, pas de lente montée vers une crise, pas de dénouement rapide. Il n'y a pas de ponctuation, pas de point de tension, pas d'alternance entre des moments plus forts et d'autres plus calmes. Certains journalistes français, lors de la présentation du film au festival de Cannes en 1988 , l'ont même considéré comme le film le plus soporifique du festival.

Cette inaction se retrouve également sur le plan esthétique. La caméra est quasi immobile. Sur un total de 362 plans, nous n'avons dénombré que 41 plans avec mouvement de caméra sur un total de 362 plans. Les personnages entrent et sortent du champ sans cette poursuite incessante de l'appareil. L'espace filmique donné à voir au spectateur n'est plus déterminé par l'action et par le récit comme le veut la tradition du cinéma classique, mais il devient un autre espace, indépendant de l'action. 


\section{L'esthétique de la non-transparence}

La seconde marque découle de la première. Étant donné la place prépondérante du récit dans le cinéma classique, il ne faut pas se surprendre que toute la mise en images et toutes les techniques et les règles de montage se soient élaborées pour mettre en valeur le récit. Par sa capacité à reproduire le mouvement, la caméra devient cette fenêtre sur le monde «réel». Et pour respecter cette impression de réel, on a développé les ressources techniques du cinéma afin de donner au récit un espace réaliste, vraisemblable et cohérent.

La conception des plans et du montage crée une impression d'unité spatio-temporelle à l'intérieur de laquelle se déroule le récit. Les raccords dans l'axe du regard ou de direction permettent aux spectateurs de se concentrer sur l'action et de la percevoir comme vraisemblable.

Le Roi des enfants s'attarde à déconstruire cette unité spatiotemporelle entre l'espace filmique et le récit. On peut classer en deux catégories les marques de cette distanciation par rapport à la représentation. Il y a les marques à l'intérieur des plans questionnant son rapport à l'espace, et il y a les marques de montage questionnant son rapport à l'espace-temps.

\section{- les marques de l'espace}

De nombreux plans du film laissent voir partiellement les corps des personnages pris entre champ et hors champ. Ces plans nous semblent fort révélateurs d'un refus d'utiliser l'espace à des fins strictement narratives.

Au tout début du film, dans le premier plan après le générique, un plan d'ensemble montre un des protagonistes en hors champ. Ce premier plan choque par ce qu'il ne montre pas. L'action, du moins la rencontre entre le chef de l'unité de travail et l'un de ses ouvriers, se déroule sur les bords du cadre, voire même à l'extérieur du champ de la caméra. Le bras, l'ombre et la fumée de cigarette, visibles dans la lumière, marquent la présence d'un personnage se situant en deçà du cadre, mais que l'on ne peut voir en entier. L'effet de frustration nous force à une prise de conscience des limites du cadre.

Cette distance face à l'espace de la représentation s'installe dès le début du film. En laissant les protagonistes dans cet espace imaginaire qu'est le hors champ, la caméra vient questionner le rôle qu'on lui attribue au cinéma classique, celui de fabriquer un espace concret pour les personnages du récit.

Tout au long du film, la caméra va poursuivre cette démarche de distanciation en laissant voir les limites du cadre. Le plan 15, par exemple, montre Lao Gan (dit La perche) en plan rapproché 
de façon à ne montrer que les jambes de sa copine placée à côté de lui. Puis, un lent mouvement de caméra vers le haut dévoile le corps et le visage de cette cuisinière. La lenteur du mouvement nous fait sentir de la caméra, son pouvoir de dévoiler ou de cacher au spectateur l'espace hors champ.

Mais la caméra se déplace rarement pour donner au spectateur un point de vue plus complet des protagonistes et de l'espace qui les entourent. Jamais n'y retrouvons-nous de travellings optiques. La caméra se fait plutôt sentir par son absence de mouvement. Comme au plan 66, l'image demeure fixe malgré la présence au premier plan d'un élève, debout, sur la gauche du cadre. Sa tête, ses jambes et son côté gauche sont laissés hors champ. Comme pour bien mettre en relief ce corps décapité, nous retrouvons Lao Gan à l'arrière-plan, bien au centre du cadre, de la tête jusqu'à la taille. Tout au long du film, les quatre côtés du cadre seront donc régulièrement investis à la frontière de leur espace concret et de leur espace imaginaire.

L'action de souligner le premier cadre par un second s'interprète réflexivement comme une marque de nontransparence. Christian Metz écrit à ce propos :

Le cadre intérieur, le cadre second, a pour effet de mettre en évidence le premier, c'est-à-dire le lieu de l'énonciation, dont il est, parmi d'autres, une «marque» fréquente et reconnaissable (...) permettant de mettre en évidence le «dispositif» du cinéma (...) (pp. 72-73).

Le plan 40 laisse voir de la fenêtre d'une des classes, pour la première fois, des enfants jouer dans la cour de l'école. Les bords de la fenêtre dédoublent le cadre de vision. Le plan 49 filme Lao Gan de l'extérieur de sa chambre. La fenêtre au centre du mur encadre bien le buste du protagoniste. Cette fenêtre souligne à neuf reprises sa présence comme cadre, et à chaque fois d'une façon différente. Le plan 321 présente une dernière fois la fenêtre en contre-jour; Lao Gan ferme le volet avant de quitter l'école. La fenêtre par laquelle Lao Gan a regardé le monde se referme, et avec elle s'amorce la fermeture du film. La fenêtre prend alors un sens particulier. L'histoire se raconte par le film et aussi à travers les yeux d'un protagoniste. En se laissant voir, les marques du travail de la caméra s'affirment en tant que regard subjectif. Elles s'énoncent comme valeur esthétique au même titre que le récit.

Une troisième catégorie de marques vient s'afficher pour mettre en valeur l'espace cinématographique par les entrées et les sorties des personnages. Dès le plan 3, Lao Gan franchit la frontière près de la caméra pour sortir du champ; et pendant ce temps, ses compagnons entrent par la porte tout au fond du décor, 
traversent l'espace et vont rejoindre Lao Gan jusqu'au hors champ pour le faire revenir dans le champ de la caméra. On fait ainsi prendre conscience au spectateur d'une importante particularité de l'espace filmique, la profondeur de champ. Contrairement au théâtre, l'espace au cinéma ne se limite pas uniquement par sa largeur mais aussi par sa profondeur, c'est-à-dire de l'objectif de la caméra jusqu'aux profondeurs de l'espace que ce dernier peut capter.

Le plan 29 en est un exemple. Lao Gan et son compagnon Heihei empruntent une petite route bordée d'arbres. Un troupeau de vaches s'avance lentement de l'arrière-plan du décor vers l'objectif de la caméra pour sortir du champ par le coin inférieur gauche du cadre alors qu'au coin inférieur droit, Lao Gan puis Heihei pénètrent dans l'espace filmique et avancent dans le décor.

L'espace filmique ne se limite donc pas aux deux côtés latéraux. Par ces entrées et sorties près de l'objectif et cette mise en valeur de la profondeur de champ, le film démontre la force esthétique et expressive propre à l'art cinématographique.

Un autre procédé utilisé pour attirer l'attention sur l'espace filmique est de faire entrer et sortir les personnages en laissant l'espace vide un certain temps. Au centième plan, après un travelling sur Lao Gan, la caméra le laisse disparaître dans la nature et le spectateur regarde ce vaste paysage esseulé un long moment. Une seconde fois au plan 121, l'image laisse le spectateur à l'extérieur de la classe alors qu'il n'y a rien d'autre à voir que le professeur qui y entre et puis qui en ressort, pour se diriger vers la gauche et sortir du cadre. Au plan 161, Lao Gan est accroupi sur un banc pendant que le directeur Chen entre et sort par la droite du cadre (on y entend le léger bruit de l'eau suggérant qu'il fait sa toilette matinale). Finalement, Lao Gan quitte par la gauche laissant le spectateur sur un décor de brume matutinale.

Au cinéma classique, l'espace n'existe que pour l'action, il doit être narrativement justifié. En laissant le champ vide, le film prend donc encore une fois ses distances face au récit.

La convention du cinéma de fiction interdit aux comédiens de regarder directement la lentille de la caméra, car elle dévoile la présence de l'appareillage ou du dispositif aux spectateurs et brise par le fait même son effet de transparence. Christian Metz écrit : «À la différence des adresses de langage, parlées ou écrites, l'adresse par le regard est réflexive.» Il écrit aussi : «Le regardcaméra introduit ainsi un retournement qui désinnocente le dispositif et le souligne d'un gros trait à l'envers (...)» (p. 40). Le récit n'est alors plus indépendant de l'acte de filmer, ni de l'acte de regarder, comme veut le laisser croire le cinéma classique. Le Roi 
des enfants utilise, à plusieurs reprises, cette rupture par le regard à la caméra comme au plan 141 où le regard de Lao Gan semble clairement prendre le spectateur à témoin. Les plans 140 et 142 sont de magnifiques plans d'ensemble extérieurs de Lao Gan, seul. La lumière orangée suggère le coucher du soleil. Entre ces deux plans, Lao Gan, en plan-buste, regarde en direction de la caméra et explique la signification du caractère «vie» (sheng) : «Vie ou vivre, c'est manger, boire. C'est pourquoi on retrouve la clef de l'eau (trois gouttes d'eau) à gauche et la "langue" à droite» (Sheng, shenghuo, huo zhe: chi, he. Suoyi you san dian shui he yi ge she).

Pour marquer sa distanciation face à cette conception classique du cinéma, le film Le Roi des enfants propose un traitement de l'espace où le travail de la caméra s'affirme comme valeur esthétique. Cette mise en présence de la caméra, des limites du cadre et de l'espace particulier au cinéma dévoile aux spectateurs l'aspect construit de l'image et de l'espace filmique. L'espace cinématographique se détermine en fonction du travail de la caméra et de son cadrage. Il n'est pas de réel mais la représentation et la construction d'une certaine réalité qui plus est, n'est pas objective. Le film se laisse ainsi voir comme acte producteur d'un système de représentation.

\section{- les marques de montage}

Les différentes marques de montage repérées dans Le Roi des enfants nous conduisent à penser le film comme instance narrative. Il résiste à se soumettre entièrement à l'obligation de créer l'illusion d'un monde réaliste, homogène et continu. Voici donc quelques particularités au niveau du montage démontrant une certaine distanciation devant l'organisation d'un monde homogène soutenant l'instance narrative.

Le cinéma classique a tendance à mener le spectateur en parfaite continuité d'un plan à l'autre. Le changement d'espace appelle la scène suivante du récit. L'espace et le temps se conjuguent en liaison avec l'intrigue. Une fois de plus, Le Roi des enfants se distingue de cette manipulation spatio-temporelle.

Il le fait dès le début, avant le générique, en nous montrant un plan de très grand ensemble. Une brume matutinale se dissipe du bas vers le haut pour laisser voir une colline sur laquelle se différencient peu à peu des bâtiments et les montagnes en arrièreplan. Le soleil poursuit sa course pour enfin se cacher derrière les montagnes. Habituellement, les plans d'ensemble au début d'un film sont là pour définir le contexte du récit dans un lieu général. Ce premier plan avant le générique aurait ainsi, au niveau narratif, une fonction de contextualisation. Il est le «Il était une fois, sur une petite colline...» 
Or, le générique vient séparer ce plan du reste du film. Les plans d'intérieur suivant le générique donnent peu d'indices d'une continuité spatiale en relation avec le paysage représenté. Toute la scène de la dernière soirée de Lao Gan avec ses compagnons de travail est tournée à l'intérieur du dortoir. Le départ de Lao Gan au petit matin se fait au bord de l'eau. Nous aurions le réflexe de combler l'espace en associant les bâtiments vus sur la colline aux lieux où s'installent les personnages, mais voilà qu'au plan 33 , Lao Gan et son compagnon Heihei atteignent cette colline après une longue marche. La colline n'abrite donc pas le camp de travail, mais l'école.

Ce premier clin d'œil fait exception à la règle de l'organisation spatiale au service du récit. On vient ainsi bousculer les habitudes acquises du spectateur en retirant au premier plan son rôle de support spatial à l'instance narrative. L'image reprend son indépendance, elle redevient un espace indéfini que l'on peut contempler et où l'on peut errer au gré du regard, du bas vers le haut, du premier plan vers le fond, de la lumière vers la noirceur, une image déchargée de sa valeur narrative, l'espace d'un moment au temps présent.

Une des séquences les plus représentatives des moyens employés dans le film pour déconstruire l'unité spatio-temporelle est la scène (en 15 plans, soit des plans 20 à 34 ) où Lao Gan accompagné de son ami Heihei quittent le camp de travail pour se rendre à l'école. Les mouvements soit de la caméra soit des personnages ainsi que le décor dans chacun des plans vont s'opposer au plan suivant, créant ainsi un effet de désorientation spatiale pour le spectateur.

Au plan 20, nos deux protagonistes quittent vers la gauche une rive sans arbre. La caméra les suit à l'aide d'un panoramique. Le plan 21 est un panoramique vers la droite sur une forêt dense et sans personnage. Ce même plan va revenir vers la fin de la séquence (plan 30) alors que logiquement, on ne peut passer deux fois dans le même espace. Le plan 22 est un plan fixe où les personnages, dos à la caméra, s'avancent sur une route bordée d'arbres. Et ainsi de suite jusqu'au plan 34. Le cinéma classique, dans la représentation d'un tel voyage, aurait davantage respecté une certaine logique de continuité en utilisant des raccords de direction dans le montage.

Nous ne pouvons traiter de montage sans aborder la question du temps au cinéma. Pour nous raconter une histoire, le cinéma classique a recours à différents procédés de montage pour évoquer le temps. Soulignons l'importance des raccords (dans l'axe, de direction, de regards, de mouvement) pour représenter la réalité 
continue du temps et de l'espace. En alternant les images de deux actions se produisant dans des lieux différents, le montage alterné permet de recréer l'impression de simultanéité de deux actions. Il est également possible d'évoquer le souvenir d'un protagoniste par des retours en arrière.

Le Roi des enfants ne cherche pas à user de ce pouvoir qu'offre le montage pour nous transporter ici et là dans le temps et dans les souvenirs des personnages. L'organisation des plans semble mettre peu d'emphase sur le temps du récit. L'expérience de Lao Gan ne s'inscrit pas dans un temps ou un espace précis et concret.

Contrairement à son application classique, l'emploi des ellipses ou coupures dans le temps ne vise pas à la «suppression des temps faibles de l'action, c'est-à-dire de ceux qui ne participent pas directement et utilement à la définition et au progrès de la séquence dramatique» (Martin, p. 256). De nombreux plans consacrés aux déplacements des personnages nous le font remarquer. Au cinéma classique, les déplacements des personnages produisent des longueurs. Des coupures, un montage condensé en quelques plans en limitent la durée. Dans Le Roi des enfants, le montage ne valorise pas cette notion d'efficacité narrative. À l'inverse, les éléments du récit se mêlent aux longs moments d'inaction et de temps morts.

Au début du film, l'arrivée de Lao Gan et de son ami Heihei à l'école prend 15 plans et dure 4 longues minutes. Au premier jour de classe, la caméra suit Lao Gan, accompagné du directeur Chen, traversant la cour pour se rendre jusqu'au local avant d'être présenté aux étudiants. Après chaque fin de cours, l'image s'attarde au fond de la classe jusqu'à ce que tous les élèves en soient sortis. Nous avons déjà décrit certains plans où, malgré le déplacement du personnage, la caméra demeure fixe laissant le champ vide et accentuant ainsi l'impression de longueur.

La scène du renvoi de Lao Gan est un autre exemple de nonrespect de cette logique d'efficacité dramatique au niveau du montage. La visite de l'inspecteur est importante, car elle conduit au renvoi de Lao Gan et au dénouement du récit. Pourtant, un seul plan est consacré à cette entrevue et de plus, celle-ci est coupée avant sa fin. Et comme pour noyer encore davantage cet instant dramatique, les deux plans avant et les trois plans après l'entrevue montrent Lao Gan traversant la cour de récréation.

Ces scènes ne cherchent-elles pas à offrir aux spectateurs l'essence même du mouvement, défini comme étant un déplacement dans l'espace et dans le temps? Quoi qu'il en soit, au cinéma, l'espace et le temps sont intimement liés. Les nombreux exemples présentés jusqu'à maintenant nous amènent à considérer 
que nous sommes davantage en présence d'une valorisation de la durée comme perception subjective plutôt que d'une mise en valeur de la reproduction du temps assujetti au récit. Gilles Deleuze s'accorde également avec cette idée que la recherche d'images cinématographiques pures (affranchies de leur soumission envers le récit) introduit dès lors un nouveau rapport au temps :

\begin{abstract}
Dans la banalité quotidienne, l'image-action et même l'imagemouvement tendent à disparaître au profit de situations optiques pures, mais celles-ci découvrent des liaisons d'un autre type, qui ne sont plus sensori-motrices, et qui mettent les sens affranchis dans un rapport direct avec le temps, avec la pensée. Tel est le prolongement très spécial de l'opsigne : rendre sensibles le temps, la pensée, les rendre visibles et sonores (pp. 28-29).
\end{abstract}

Tel qu'évoqué par Deleuze, le son joue également un rôle important dans l'esthétique du montage. Le son d'ambiance et la parole, nécessairement présentés en temps réel, participent pour beaucoup à la perception de la continuité spatio-temporelle en se juxtaposant à la bande image qui elle, malgré ses raccords dans l'axe, demeure fragmentée et discontinue.

Longtemps confiné aux textes idéologiques, le cinéma chinois a très peu utilisé le potentiel expressif que pouvait offrir la sonorisation. Les pauvres effets sonores se réduisaient trop souvent à une utilisation abusive d'une musique stéréotypée et redondante par rapport aux images. Comme pour l'image, Le Roi des enfants cherche à se distinguer des productions passées. Ainsi, l'univers sonore du film propose une esthétique propre au cinéma en démontrant la puissance expressive non seulement des images mais aussi du son.

Dans un premier temps, il est important de noter qu'il n'y a pas de musique en son off. On a complètement éliminé le thème musical qui accompagne habituellement le générique du film. Pendant que les noms de la distribution apparaissent à l'écran, on entend seulement le grattement d'une craie sur un tableau noir. Les seules mélodies dans le film sont des chansons qui prennent clairement leurs sources dans l'espace de la diégèse. L'espace sonore saturé par une musique lyrique de violons et de tambours est ainsi remplacé par des sons naturels et familiers pour le spectateur chinois.

Au plan 80 débute une séquence où les sons in intensifiés créent un univers subjectif et esthétique proprement cinématographique. Après avoir réalisé qu'il n'y a qu'un seul livre pour toute la classe, le professeur s'installe au tableau pour copier le texte de la leçon pendant que les élèves se mettent eux aussi à recopier les 
caractères. Au plan 82 , les bruits de grattement des crayons et de la craie sur le tableau se font entendre jusqu'au plan 86 où ils s'amplifieront graduellement pour durer jusqu'au plan 87 . Ce dernier montre la jeune chef de classe qui dépose son crayon sur le bureau et regarde en direction du hors champ. Le plan 88 laisse voir la cour de récréation vide, vue du cadre de la porte. Le grattement des crayons s'est arrêté, le silence règne. Puis, l'image du plan 89 retourne dans la classe où les élèves sont encore en train de copier. Les sons amplifiés de la copie reprennent jusqu'au plan 90 où ils diminuent alors peu à peu jusqu'au silence. Le professeur tient dans sa main une lampe à huile; on s'aperçoit que la nuit est tombée. Les enfants fatigués ont cessé de copier. Le bruit des criquets se fait entendre du plan 91 au plan 95 . La séquence se termine avec le souffle du professeur éteignant sa lampe. L'alternance entre les sons in amplifiés et le silence ajoute aux images une intensité subjective qui n'aurait pu être obtenue avec la musique.

Le film de Chen Kaige se démarque encore une fois d'un certain classicisme au cinéma par l'emploi fréquent de la noncorrespondance du son et de l'image. La stratégie la plus souvent observée consiste à placer en son hors champ les paroles des protagonistes libérant ainsi l'image de son rôle de support spatial pour le protagoniste parlant. La scène où Lao Gan en visite chez ses copains du camp de travail raconte sa nouvelle vie à l'école en est un exemple probant. Une discussion animée s'amorce à partir du fait qu'il n'y a pas de dictionnaire à l'école. Soudain, un plan rapproché (le plan 172) du vieux chef d'équipe en train d'allumer sa pipe à eauet qui semble bien peu préoccupé par le sujet de la conversation est enchâssé à travers les images des jeunes qui discutent. Cette simple image de la traditionnelle pipe à eau accaparant toute l'attention du vieil ouvrier des montagnes, juxtaposée au discours des jeunes sur l'importance du dictionnaire (symbole de la connaissance), évoque par métaphore le choc des cultures entre la jeune génération citadine intellectuelle et la génération paysanne qui a si bien su se passer d'éducation jusqu'à ce jour.

Plus loin, au plan 226, Lao Gan quitte le tableau sur lequel les caractères «Shang Xue» (aller étudier) sont écrits. La caméra reste fixe devant ces caractères pendant qu'en son hors champ se poursuit la leçon : «Désormais, il faut, premièrement, écrire les caractères lisiblement. Que vos caractères ne soient pas beaux n'a pas d'importance.»

Mais le moment le plus significatif reste la dernière séquence qui marque la fin du film. Elle s'amorce au plan 327 au moment 
où, sur le chemin du retour, Lao Gan passe à nouveau par la colline aux souches brûlées vue au début du film. À partir du plan 329 , le son léger des cloches à vaches se distingue peu à peu du reste des sons d'ambiance (criquets et oiseaux). Le son des cloches s'intensifie pendant que les images ne laissent voir aucune vache. On montre alors au plan 333 le petit berger en train d'uriner sur l'une des souches; puis au plan 334, un gros plan de la tête du berger caché par un chapeau percé. Puis, des plans 335 à 350, un montage rapide enfile une variété de prises de vue sur les souches brûlées aux formes insolites pendant que le bruit retentissant des cloches à vaches envahit tout l'espace sonore. Le tumulte s'estompe peu à peu pour retourner au calme des sons de criquets pendant que le montage des images reprend lui aussi son rythme lent pour un dernier échange de regards entre le jeune berger et Lao Gan dans ce décor dévasté.

Les différents procédés que nous venons de décrire démontrent que le film Le Roi des enfants s'éloigne d'une exploitation classique des éléments sonores qui ne sont trop souvent qu'une redondance de l'information avec les éléments visuels. Le fait de ne pas recourir à la musique en son off montre bien qu'un film n'a pas besoin d'ajouter, à l'atmosphère et au rythme déjà établis par le montage, un thème musical en fond sonore. Par la noncorrespondance des sons et des images, les dialogues s'inscrivent comme valeur expressive tout autant que les autres éléments sonores qui tantôt in, tantôt hors champ et parfois même off, évitent non seulement une certaine forme de redondance mais redonnent aux éléments tant visuels que sonores leurs qualités expressives. La juxtaposition des sons et des images participe à la création d'une esthétique proprement cinématographique.

Dans son opposition au classicisme, le cinéma moderne non seulement dévoile les marques du travail cinématographique que le cinéma classique, au contraire, cherche à effacer, mais aussi les considère-t-il comme faisant partie intégrante de la représentation. Les plans longs et fixes, l'espace vide, la caméra dévoilée par le regard de l'acteur, la profondeur de champ, le redoublement du cadre, le montage-cut sont les marques d'un cinéma qui s'affirme désormais comme un art de l'espace et du temps offrant un univers visuel et sonore expressif et subjectif tout à fait spécifique.

Cette forme cinématographique, nouvelle pour les Chinois, force le spectateur à s'impliquer dans la construction du film dont les images désormais ne suivent plus la simple logique du récit. Les plans immobiles et les images anodines forcent l'attention du spectateur qui doit donner un sens (image lisible selon Deleuze) à cet espace visuel et sonore, car les signes sonores sont tout aussi 
importants que les signes-images. Le spectateur, ainsi engagé dans le processus de construction du sens, pourra suivre un certain cheminement réflexif enchâssé dans le film. Comme si l'on assistait tout à coup à un renversement des rôles où le récit se place au service de l'expression cinématographique.

Ainsi, tout au long du film, des plans longs et souvent immobiles viennent s'intercaler les uns dans les autres nous faisant lentement sentir se développer la pensée du film. On se souviendra de ces nombreux plans de Lao Gan, seul, regardant par la fenêtre ou en train d'explorer son environnement. On retiendra également le long plan silencieux du miroir brisé dévoilant, dans la pénombre de la nuit, le double reflet de Lao Gan (plan 109), le plan quelque peu insolite du veau venant rôder près de l'abri du professeur (plan 195) et encore celui du lent panoramique vers le bas montrant un vieil arbre, un bâtiment puis un bol vide (plan 236). On se souviendra également du message de Lao Gan, «... ne copie plus jamais, pas même le dictionnaire», inscrit sur la souche ainsi que le caractère «Bœuf-Eau» laissé sur le tableau de la classe. Ces plans immobiles (caméra et / ou objet filmé) montrant l'unique, le solitaire, intercalés à travers les plans d'ensemble (élèves, amis) dévoilent l'être dans son détachement du groupe. Un seul arbre par opposition aux bâtiments, un veau par opposition au troupeau de vaches, Lao Gan devant les mots du dictionnaire, le petit berger illettré par opposition aux souches brûlées.

Ces plans dévoilent un cheminement vers la distanciation, la prise de conscience de soi, la remise en question de son rapport au groupe et à la culture. Ce cheminement laisse supposer une certaine conclusion : l'Homme ne doit pas être au service du maintien d'une culture au service de la stabilité sociale; c'est la culture qui doit être au service de l'Homme. L'être humain doit se réapproprier le langage pour le mettre au service de l'expression de sa propre expérience.

À la fin du film, les souches brûlées aux formes parfois humaines sur lesquelles urine le jeune berger illettré suggèrent une culture toujours bien enracinée mais sans vie. Sans le savoir, le survivant est celui qui s'en moque et qui poursuit librement sa route comme les vaches que l'on entend en son hors champ et qui, librement, boivent l'urine salée malgré les idées bien pensantes et cultivées de la société. Telle est l'idée s'exprimant sous les traits du caractère «Bœuf-Eau».

Se juxtapose ainsi une double lecture du film. Le niveau cinématographique et le niveau narratif proposent un même cheminement résolument moderne, du moins pour l'Occident. Ils proposent une distanciation face à la tradition amenant à la 
conscience de soi et à un questionnement de soi dans son rapport à la tradition. Son émancipation, celle de l'homme et du cinéma, passe par une réappropriation du langage au service de l'affirmation de son propre potentiel expressif.

\section{Université du Québec à Montréal}

\section{NOTE}

1 Définition de Jean Baudrillard dans Encyclopaedia Universalis, corpus 12 (Paris : 1985) p. 424.

\section{OUVRAGES CITÉS}

Deleuze, Gilles. Cinéma 2: L'Image-temps. Paris : Minuit, 1985. Martin, Marcel. Le Langage cinématographique. Paris : Cerf, 1977. Metz, Christian. L'Énonciation impersonnelle ou le site du film. Paris : Méridiens Klincksieck, 1991.

\section{GLOSSAIRE DES PRINCIPAUX TERMES CHINOIS (par ordre alphabétique)}
A Cheng
阿城
Chen Kaige
陳凱歌
Haiziwang
孩子王
Heihei
黑黑
Lao Gan
老杆
niu + shui
牛十水
shang xue
上學
sheng
生
sheng, shenghuo, shengzhe :
生、生活、生者：
chi, he suoyi you sandian shui
吃、喝所以有三點水
he yige she
和一個舌
zhiqing
知青 